\title{
Investigation of Vortex Development during Polymer Melt Flows by Flow Birefringence
}

\author{
Jan Musil $^{\mathrm{a}}$, Martin Zatloukal ${ }^{\mathrm{a}}$, Tim Gough ${ }^{\mathrm{b}}$ and Mike Martyn ${ }^{\mathrm{b}}$ \\ ${ }^{a}$ Centre of Polymer Systems, Polymer Centre, Tomas Bata University in Zlin, \\ nam. T. G. Masaryka 5555, 76001 Zlin, Czech Republic \\ ${ }^{b} I R C$ in Polymer Engineering, School of Engineering, Design and Technology, University of Bradford, \\ Richmond Road, BD7 1DP, Bradford, United Kingdom
}

\begin{abstract}
The vortex development of low-density polyethylene Lupolen $1840 \mathrm{H}$ polymer melt under various processing conditions has been investigated by flow induced stress birefringence technique. It has been revealed that effect of mass flow rate and temperature on the vortex size has non-monotonic character, which is in good agreement with laser-Doppler velocimetry based measurements reported for the same polymer in the open literature. This suggests that the flow induced stress birefringence technique can be considered as the quick and reasonably precise tool for vortices shape/size visualization and quantification at the slit die entry region.
\end{abstract}

Keywords: Vortex formation, Secondary flows, Flow instabilities, Flow birefringence, Planar extensional viscosity, Extrusion.

PACS: 47.20.Gv， 47.20.Lz， 47.32.-y，47.50.-d， 47.50.Ef， 47.50.Gj，47.57.Qk，47.80.Jk, 83.60.Wc, 83.80.Sg, 83.85.Ei

\section{INTRODUCTION}

"In 1866 I made some attempts to ascertain whether the state of strain in a viscous fluid in motion could be detected by its action on polarized light." This sentence written by James C. Maxwell [1] in 1873 meant the very beginning of flow birefringence. Through many decades, this technique has been improved and now it represents a powerful tool for investigation mainly unstable and unwanted polymer melt flow phenomena [2-5].

One of these phenomena occurring in the extrusion of polymer melts is the generation of vortices, where the melt rotates very slowly. The residence time of the macromolecules in these zones is very long and therefore the molecules can degrade [6-9]. Thus, from the processing point of view, the polymer as well as the processing conditions and the die design should be chosen carefully to avoid this undesirable phenomenon. To optimize the manufacturing processes, the understanding of the reasons for the vortex development during the flow of polymer melts is necessary.

However, the experimental visualization study of vortices in the slit contraction geometries is really difficult task. The difficulties can be explained in the following way. Vortices are generated by rotating particles (on the molecular level by polymer macromolecules), thus velocity field is the crucial quantity to measure and study.

Novel Trends in Rheology IV

AIP Conf. Proc. 1375, 14-25 (2011); doi: 10.1063/1.3604465

(C) 2011 American Institute of Physics 978-0-7354-0935-4/\$30.00 
Moreover, in the entry region of the slit die (which is necessary to use for visualization purpose), the velocity field has three-dimensional nature [10], which means that the velocity is changed across the length and also the width of the slit. Measurement of such complex velocity field can be reached by two main techniques: laser-Doppler velocimetry (LDV) $[11,12]$ or particle tracking velocimetry (PTV) [13].

The first, LDV, measures direction and speed of any particle through two laser beams which are intersected in the focal point of a laser where they interfere and generate a set of straight fringes. As particle passes through the fringes, it reflects light and by measuring the Doppler frequency-shift of the scattered light is possible to calculate the direction and velocity of the tracer particle. Although, this measurement is very precise, it is also very time-consuming and as the consequence of this, study material can thermally degrade inside the vortices which can be possible source of error especially in the case of polymer melts with low level of thermal stability.

The second, PTV, is based on adding small quantities of micro-sized glass beads into the hopper of the extruder and mixed with the bulk polymer to provide isokinetic seeding of the flow. Then, these beads in the slit extrusion die visualize track for each streamline. With utilization so-called "laser-sheet" can be visualized and then recorded velocity field for different plane inside the die. This technique is also time-consuming and another problem consists in deformation of real streamline by the glass bead mass.

From this brief overview of velocity field measurement techniques it is clear, that the main problem is long testing time. Therefore, the main aim of this paper is to investigate if polymer melt flow induced stress birefringence, which actually visualizes stress not velocity, is possible to use for quick and sufficiently precise determination of vortex shape/size in entry region of the slit visualization extrusion die.

\section{EXPERIMENTAL}

Experimental investigation of mass flow rate/temperature influence on vortex shape/size in entry region of slit die was performed for LDPE Lupolen $1840 \mathrm{H}$ (Lyondell Basell, Germany, basic characteristics are summarized in [12]) polymer melt on special flow birefringence extrusion line which is in detail described below also with chosen experimental processing conditions.

\section{Experimental Setup}

The principle of flow birefringence measurement [14-17] can be explained with the help of plane polariscope. The polarizer converts the source light into plane polarized light. When monochromatic polarized light enters specimen (polymer melt flow in the visualization cell), it is refracted into two plane polarized components of which velocities are dependent on refractive index of specimen. Then the analyzer resolves the components into one plane so that the effect produced by the orientation in specimen can be measured from the resulting interference waves. Two distinct fringe patterns can be observed, isoclinic and isochromatic. Isoclinic fringes are produced by plane polarized condition whereas isochromatic ones by circular polarized condition. 
The overall 3D view of the Bradford experimental flow visualization setup is depicted on Figure 1. It is consisted from conventional single screw extruder Betol BK38 (Davis-Standard, UK) with diameter of $38 \mathrm{~mm}$ and length of 30D heated by four electrically powered zones, video recorder for recording experimental birefringence movies on analog tape (25 frames per second), two computers for controlling extruder and transfering birefringence movies from analog to digital (through BT848 based PC-TV card), respectively and optical bench moveable in all three dimensions.

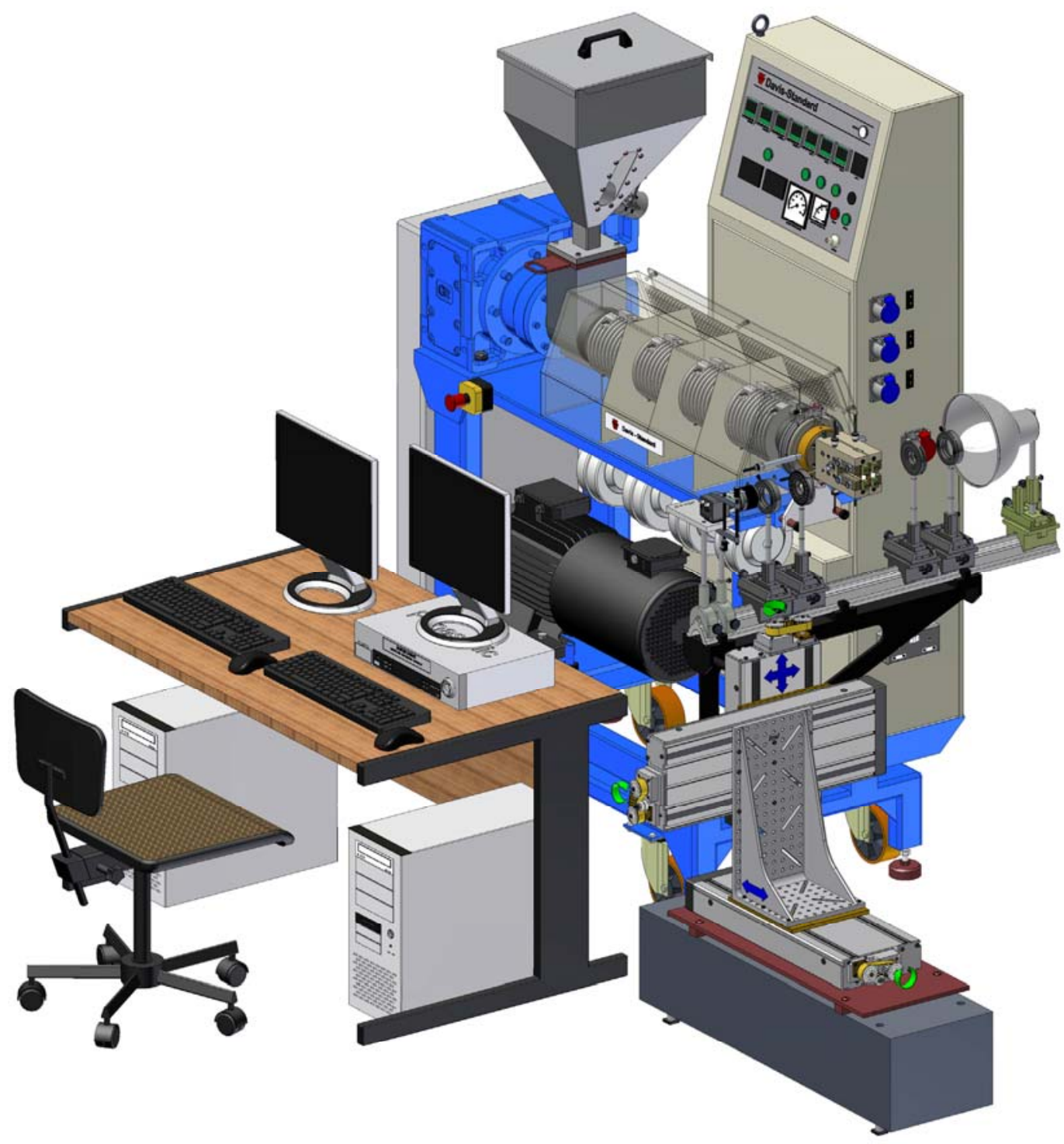

FIGURE 1. Overall 3D view of the Bradford experimental flow visualization extrusion setup (in scale). 
Detail schematic view of the optical bench is described in Figure 2. From right to left is consisted of light source (mercury vapour lamp with wavelength $\lambda=546 \mathrm{~nm}$ ), polariser plate, two quarter wave plates (between them is placed flow visualization cell), analyser plate, colour filter and CCD analog video camera (Pulnix PEC 3010 model with resolution of $720 \times 576$ pixels) equipped by Nikon lens and extension tube of $15 \mathrm{~mm}$ in length.

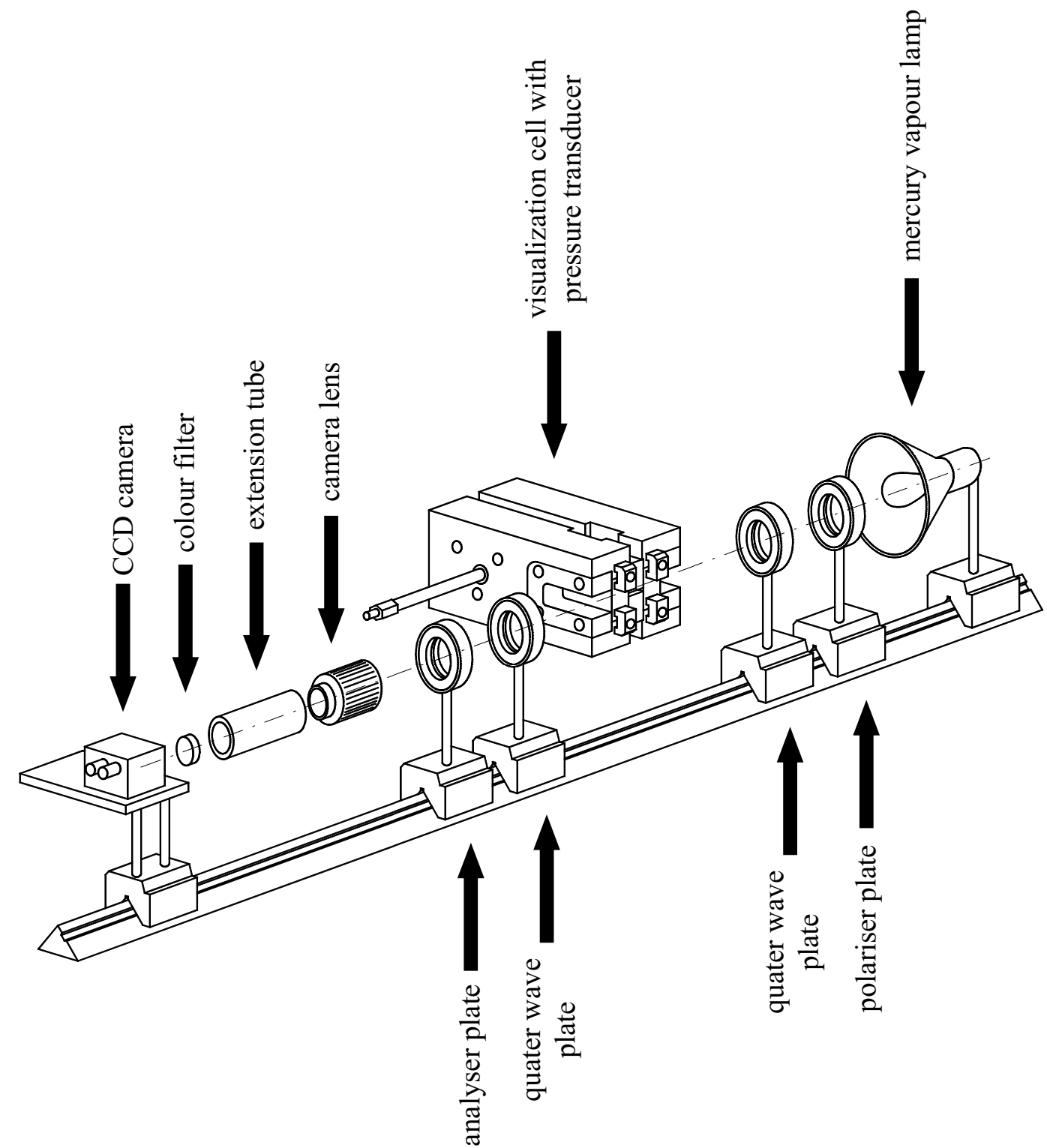

FIGURE 2. Schematic view of optical bench for flow visualization experiments (not in scale).

The flow visualization cell is made as universal slit die with strain-free borosilicate glass windows placed on either sides of the flow visualization cell to observe flow behaviour of polymer melt in the entrance region of the die, one pressure transducer in 
range of 1,500 PSI (10.3421 MPa), and rectangular slit flow geometry formed by two inside-die steel inserts. The detail of the flow geometry used in all our measurements is shown in Figure 3. Contraction ratio is $16: 1$ and the dimensions are following: upstream channel depth $H=15 \mathrm{~mm}$, slit width $\mathrm{W}=10 \mathrm{~mm}$, slit gap $\mathrm{G}=0.9375 \mathrm{~mm}$ and slit length $\mathrm{L}=15 \mathrm{~mm}$.

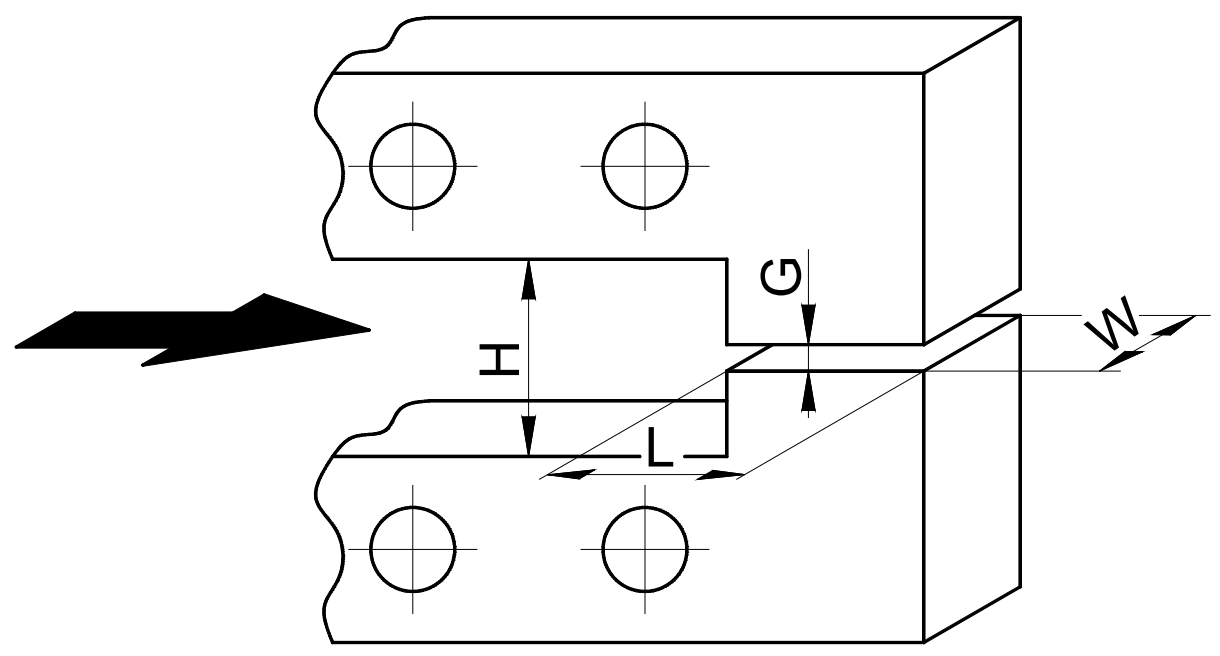

FIGURE 3. Schematic view of two inside-die steel inserts determining flow geometry (not in scale).

The experimental investigation was carried out under following conditions. Extruder zones were heated on temperatures (from the hopper to the die): $T_{1}=150^{\circ} \mathrm{C}$, $T_{2}=160^{\circ} \mathrm{C}, T_{3}=160^{\circ} \mathrm{C}$ and $T_{4}=160^{\circ} \mathrm{C}$, respectively. Two transition parts between extruder and flow visualization cell as well as flow visualization cell were stepwise heated on $135^{\circ} \mathrm{C}, 130^{\circ} \mathrm{C}$ (die temperature of $130^{\circ} \mathrm{C}$ ), $150^{\circ} \mathrm{C}, 150^{\circ} \mathrm{C}$ (die temperature of $150^{\circ} \mathrm{C}$ ), $162^{\circ} \mathrm{C}, 170^{\circ} \mathrm{C}$ (die temperature of $170^{\circ} \mathrm{C}$ ), $170^{\circ} \mathrm{C}, 190^{\circ} \mathrm{C}$ (die temperature of $190^{\circ} \mathrm{C}$ ), respectively. Flow visualization experiments were performed as 15 minutes tests at different mass flow rates ranging from $0.04 \mathrm{~kg} . \mathrm{hr}^{-1}$ up to $1.7 \mathrm{~kg} . \mathrm{hr}^{-1}$ (i.e. from $10 \mathrm{~s}^{-1}$ up to $440 \mathrm{~s}^{-1}$ in terms of apparent shear rates). These rates varied according to investigated temperature and it should be finally mentioned that the maximum apparent shear rate was chosen close under appearance of well-known melt fracture instability.

\section{Results and Discussion}

As the first, validation of vortex shape determined from stress birefringence measurement was performed. The result of this procedure is shown in Figure 4. On the right hand side is depicted real birefringence image composed from 58 single images (taken from the experimental birefringence movie in different duration times). In each image the particle in entry region is slightly moved, thus whole track for this particle can be viewed. The movement of the particle is firstly, on the vortex boundary, then one rotation inside the vortex and finally pushing out of the vortex to the slit. It is clearly seen that the streamline of such particle (moving along the vortex 
boundary) closely trace the shape of stress fringes. As introduced above, such mass particle can deform the streamline and also its position in width of the die is not exactly known. Another limitation is integral nature of flow birefringence measurement, which means that stress fringes are the summations of stresses in different vertical planes of the visualization cell. However, if these possible errors are kept in mind, this technique is much faster than other methods mentioned in the introduction section.

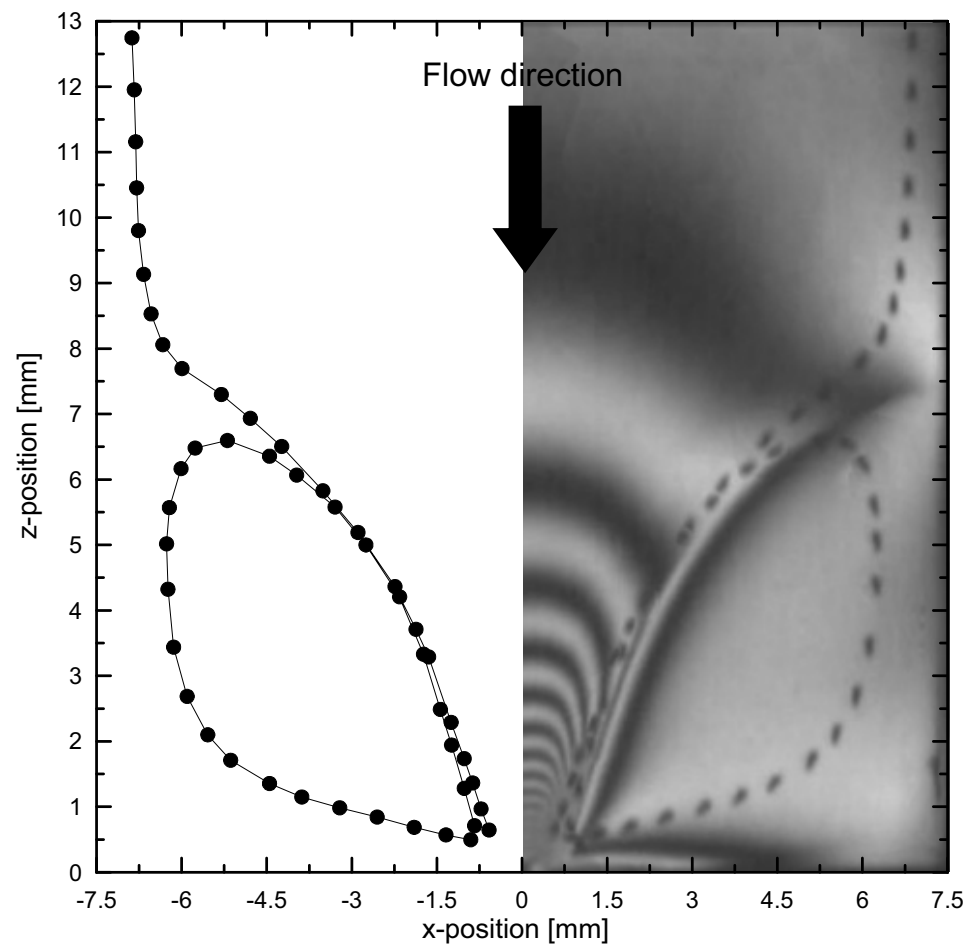

FIGURE 4. Particle tracking analysis during extrusion of LDPE Lupolen $1840 \mathrm{H}$ polymer melt at $190^{\circ} \mathrm{C}$, mass flow rate of $0.88 \mathrm{~kg} \cdot \mathrm{hr}^{-1}$ and corresponding apparent shear rate of $222 \mathrm{~s}^{-1}$ (left hand side digitized particle tracking in Didger ${ }^{\mathrm{TM}}$, right hand side - real stress birefringence field image with one particle in different duration times).

After this positive verification, all other vortex shapes and sizes have been digitized and calculated only from the flow birefringence images. For each processing condition, last frame (image) from each experimental movie was taken, then it was calibrated through four corner points and finally vortex shape was digitized and its size was calculated in special software Didger $^{\mathrm{TM}}$ (Golden Software, USA). This procedure was repeated three times in order to eliminate "human error" in the creation of vortex shape by manually "clicking". Some examples of this procedure are shown in Figures 5 and 6.

Firstly, in Figure 5 vortex shapes/sizes for different mass flow rates (MFR) at one processing temperature $\left(170^{\circ} \mathrm{C}\right)$ are shown. It can be seen that the vortex area at increasing MFR firstly increasing and then decreasing. Secondly, the same trend, visible more pronounced, is shown in Figure 6 where, on the contrary with previous example, same MFR is kept and temperatures are changed from $130^{\circ} \mathrm{C}$ up to $190^{\circ} \mathrm{C}$. 

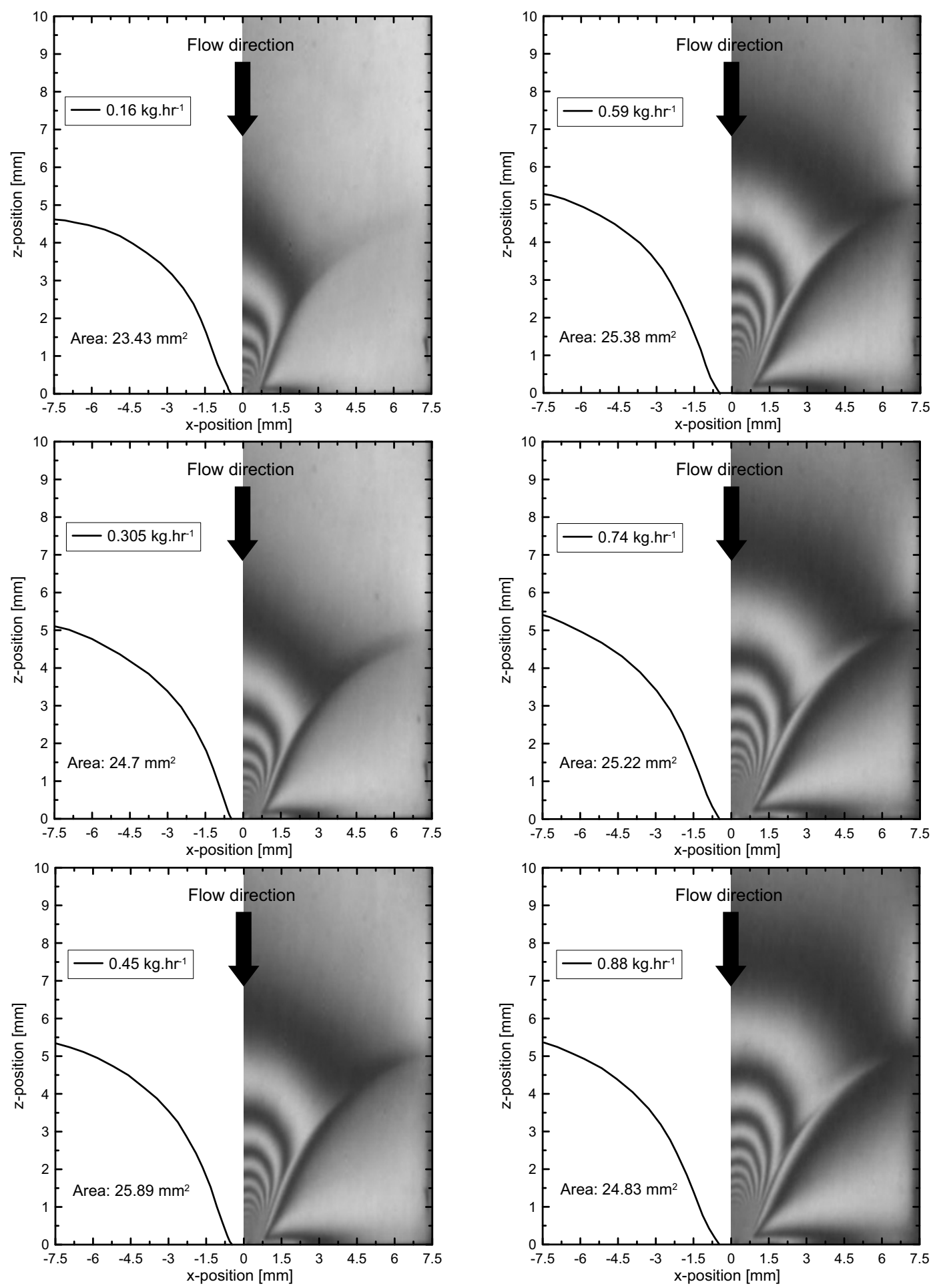

FIGURE 5. Visualization of the vortex boundaries for LDPE Lupolen $1840 \mathrm{H}$ at $170^{\circ} \mathrm{C}$ at different mass flow rates (in each graph, left hand side represents vortex boundary digitized from stress field, right hand side presents image of real flow birefringence stress field, respectively). 

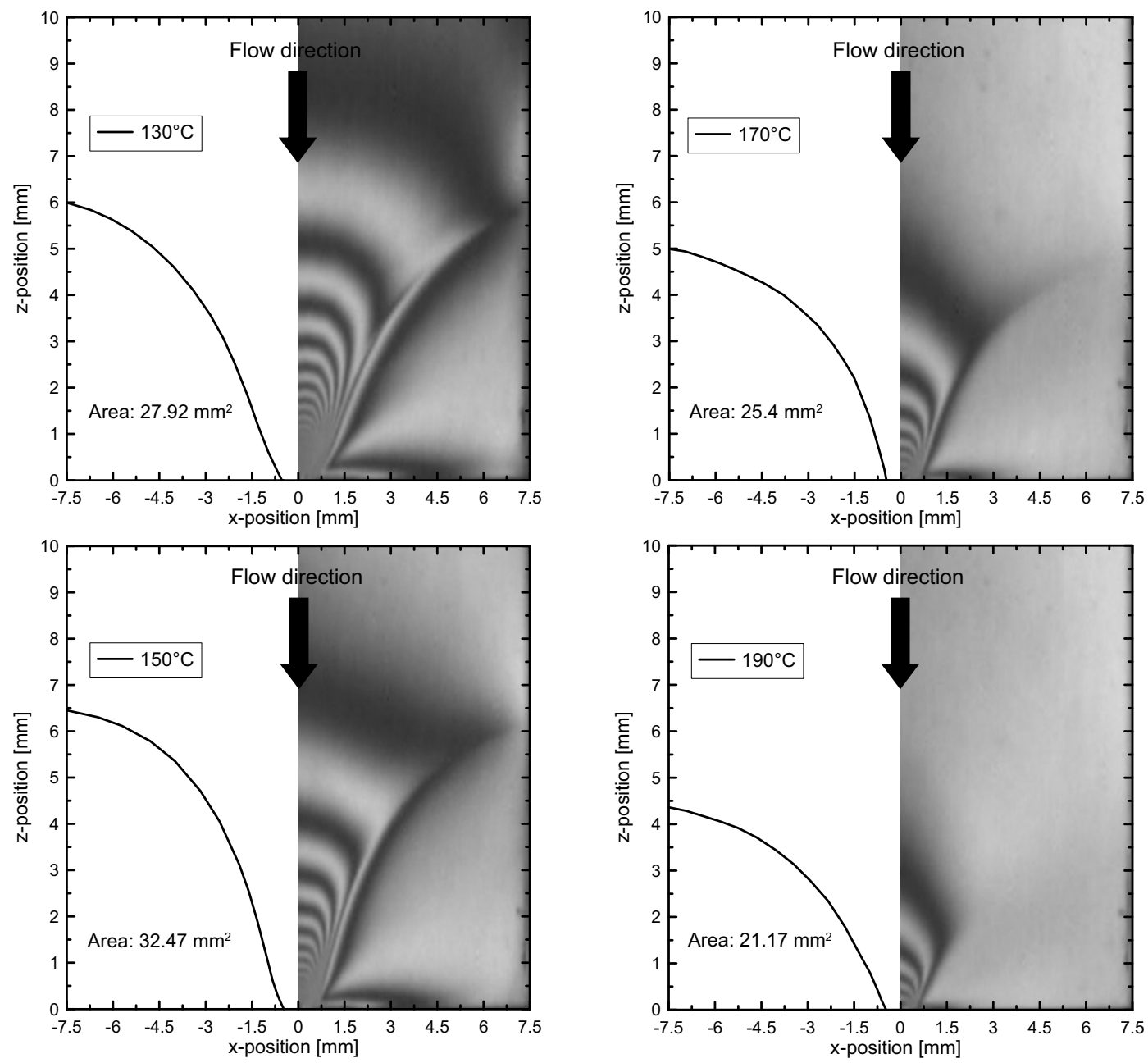

FIGURE 6. Visualization of the vortex boundaries for LDPE Lupolen $1840 \mathrm{H}$ at mass flow rate of $0.15 \mathrm{~kg} . \mathrm{hr}^{-1}$ for different processing temperatures (in each graph, left hand side represents vortex boundary digitized from stress field, right hand side presents image of real flow birefringence stress field, respectively).

\section{Effect of Mass Flow Rate on Vortex Size Development}

In order to better visualize the trends in vortex sizes, areas of digitized vortex shapes were plotted as functions of mass flow rates. The results are shown in Figures 7-10 and they can be described and explained in the following way. In all graphs vortex sizes are non-monotonic dependence of MFR (runs through local maximum). These dependences can be attributed to the non-monotonic behavior of extensional rate dependent planar extensional viscosity (well-known strain hardening). Similar results can be also found in open literature for the same polymer melt [12]. 


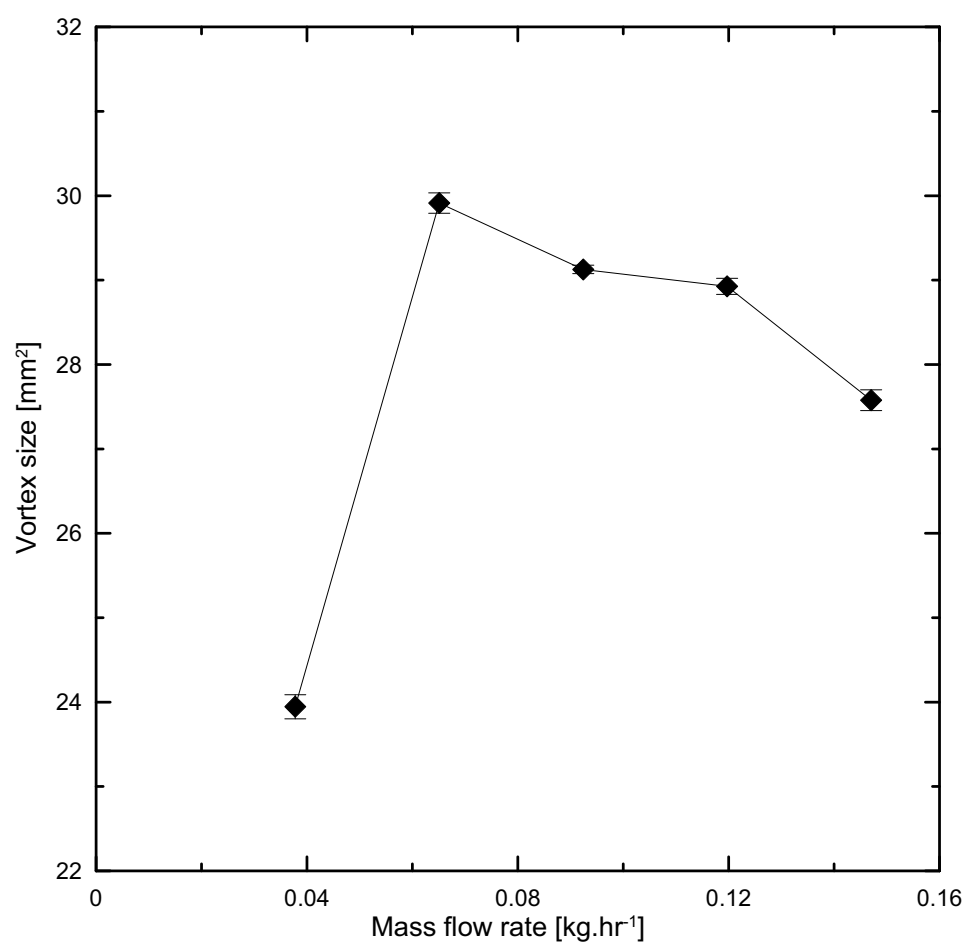

FIGURE 7. Visualization of the vortex size as the function of the mass flow rate during extrusion of LDPE Lupolen $1840 \mathrm{H}$ polymer melt at $130^{\circ} \mathrm{C}$.

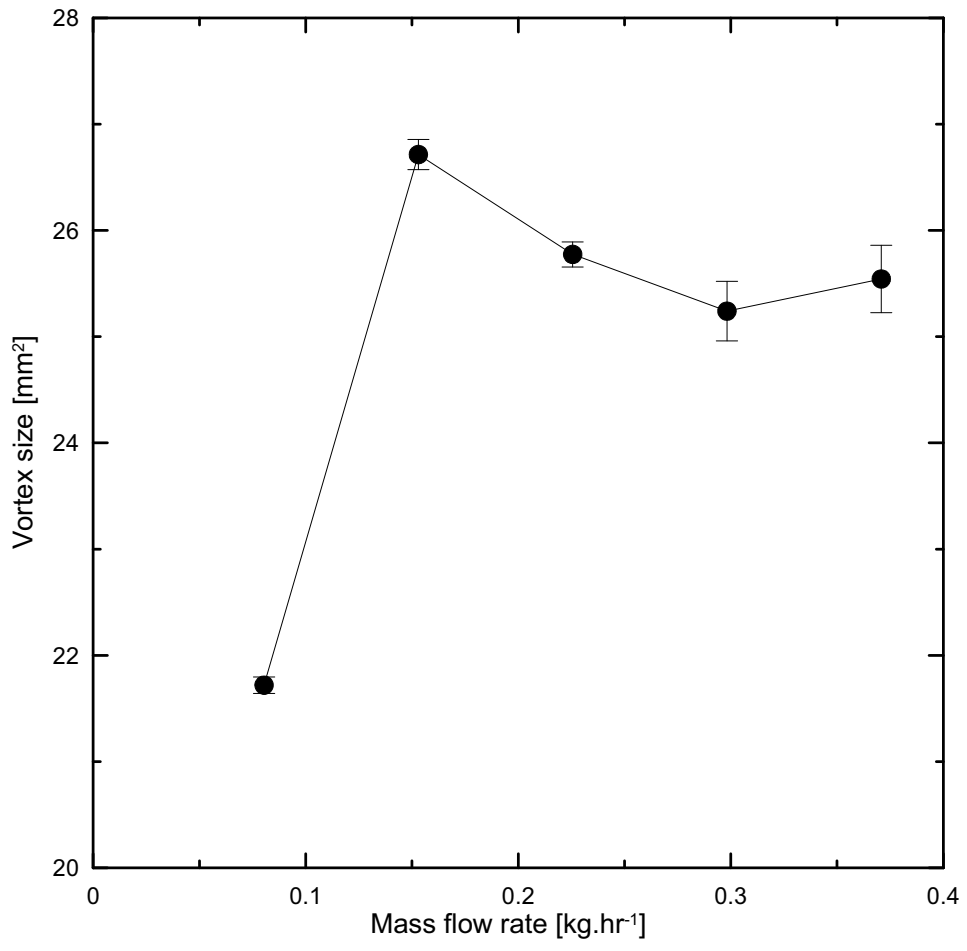

FIGURE 8. Visualization of the vortex size as the function of the mass flow rate during extrusion of LDPE Lupolen $1840 \mathrm{H}$ polymer melt at $150^{\circ} \mathrm{C}$. 


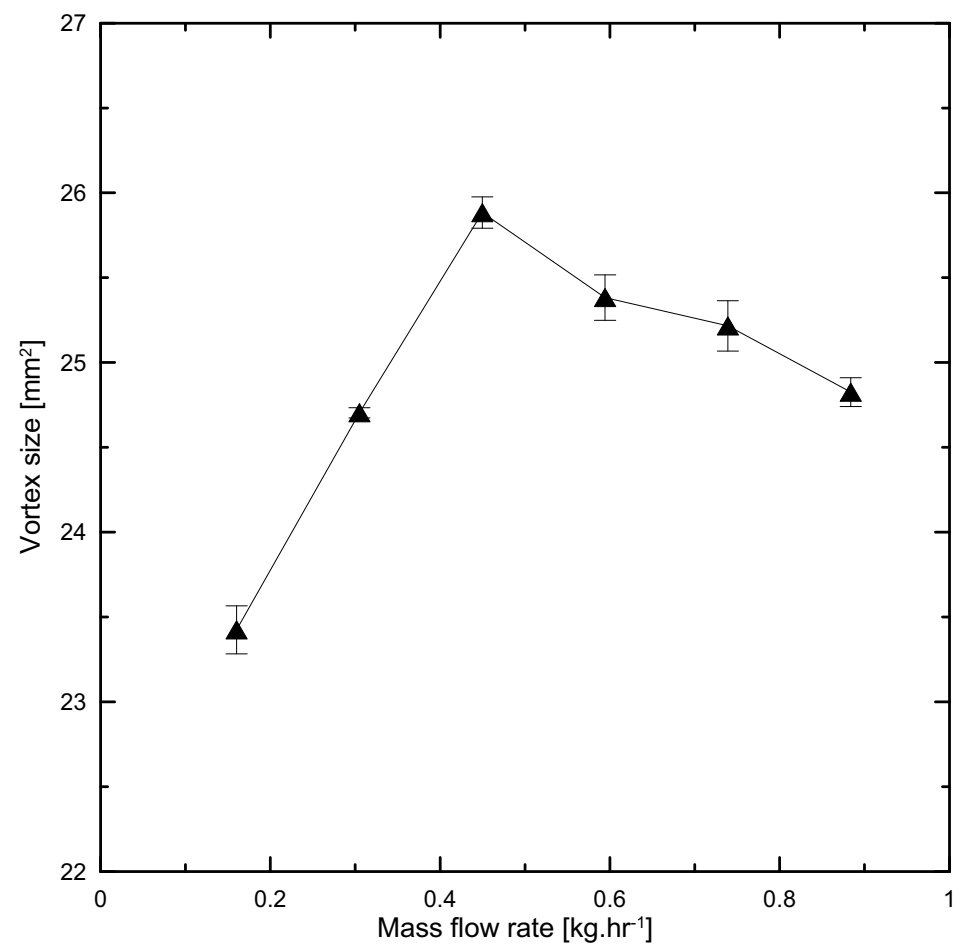

FIGURE 9. Visualization of the vortex size as the function of the mass flow rate during extrusion of LDPE Lupolen $1840 \mathrm{H}$ polymer melt at $170^{\circ} \mathrm{C}$.

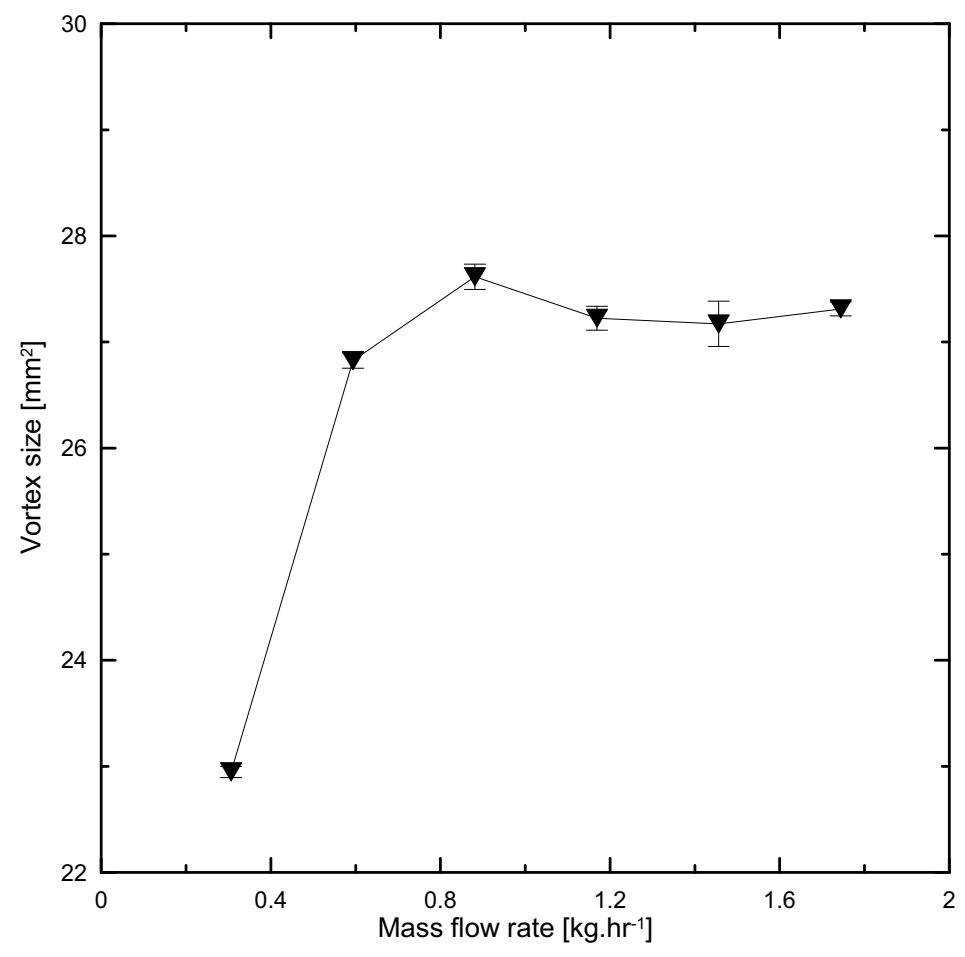

FIGURE 10. Visualization of the vortex size as the function of the mass flow rate during extrusion of LDPE Lupolen $1840 \mathrm{H}$ polymer melt at $190^{\circ} \mathrm{C}$. 


\section{Effect of Temperature on Vortex Size Development}

Temperature dependent vortex size development is shown in Figure 11. In this graph, data taken from open literature measured by precise laser-Doppler velocimetry technique [12] are also included to compare with our results. It should be noted that the error bars in literature's data represent three independent measurements, but in our case they only mean three independent shape digitalization procedures. Thus, our error bars are more close to the average value.

From the comparison of both data sets it is clearly seen that the trend is the same. Thus, firstly size increases, reaches maximum and then decreases. Some differences can be explained by slightly different contraction ratio (14:1 in [12], 16:1 in this work) and also slightly different slit die dimensions.

The non-monotonic vortex size development can be explained by non-monotonic temperature dependent planar extensional viscosity. In this dependence, maximum moves from low extensional strain rates to higher ones for increasing melt temperatures. This seems to be the driving mechanism for the maximum appearance in the vortex size vs. temperature dependence.

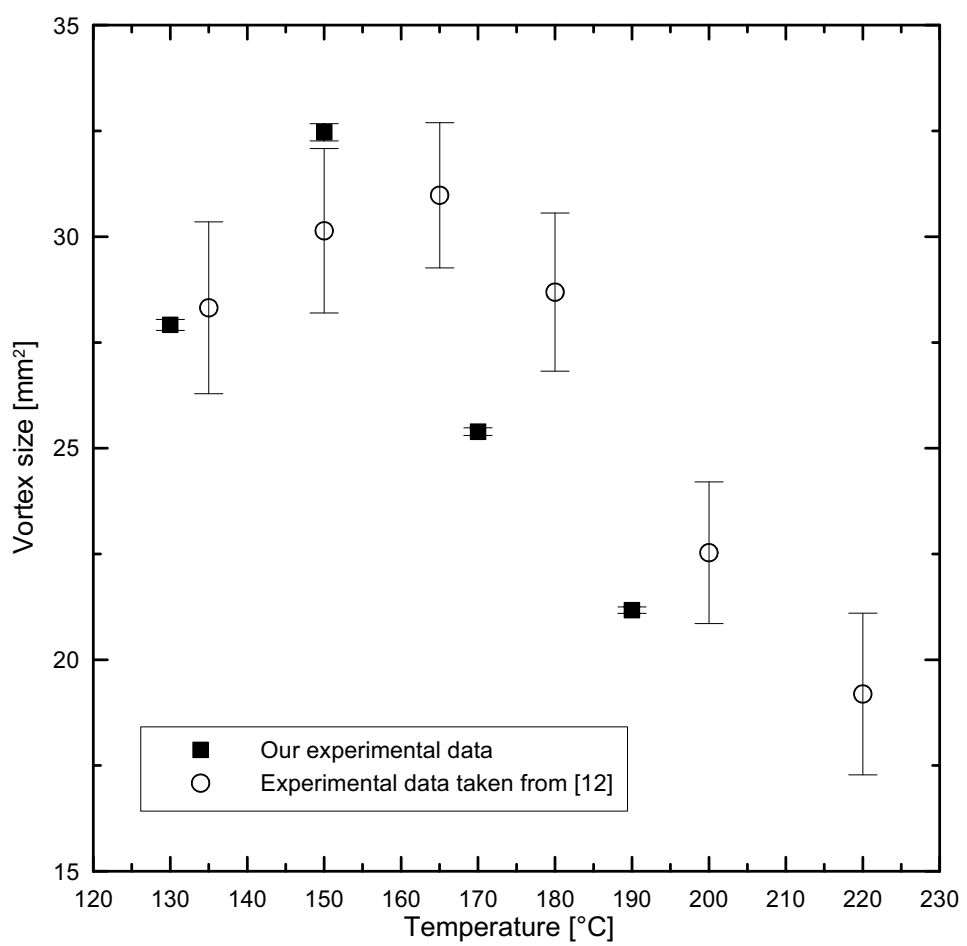

FIGURE 11. Visualization of the vortex size as the function of the temperature during extrusion of LDPE Lupolen $1840 \mathrm{H}$ polymer melt at fixed mass flow rate $\left(0.15 \mathrm{~kg} \cdot \mathrm{hr}^{-1}\right)$. Comparison with the results from open literature is also included.

This preliminary study investigates new methodology for vortex shape/size evaluation. The trends obtained from this technique are similar as published in open literature [12] for the same material, similar slit die contraction ratio and processing conditions. Thus, with keeping some limitations in mind, flow induced stress 
birefringence technique can be considered as quick and sufficiently precise method for evaluation of vortices in entry regions, especially for thermally non-stable polymer melts where short testing time is the crucial condition.

\section{CONCLUSION}

It has been demonstrated that stress birefringence technique can be used for the vortex shape/size determination. It has been found that the vortex size primarily increases, reaches a maximum and then it decreases again with increasing temperature and mass flow rate, which can be attributed to the non-monotonic behavior of the planar extensional viscosity for the branched LDPE polymers. Moreover, these results are in good agreement with data published in open literature.

\section{ACKNOWLEDGMENTS}

This work has been supported by the internal grant of TBU in Zlín No. IGA/3/FT/10/D funded from the resources of specific university research.

This article was written with support of Operational Program Research and Development for Innovations co-funded by the European Regional Development Fund (ERDF) and national budget of Czech Republic, within the framework of project Centre of Polymer Systems (reg. number: CZ.1.05/2.1.00/03.0111).

Finally, authors thank Steve Brook, Ian Smith and Roy Dixon from University of Bradford for technical support during flow visualization experiments.

\section{REFERENCES}

1. J. C. Maxwell, Roy. Soc. Proc. XXII. 22, 46-47 (1873).

2. J. P. Tordella, J. App. Polym. Sci. 7, 215-229 (1963).

3. T. F. Ballenger et al., Trans. Soc. Rheol. 15, 195-215 (1971).

4. J. M. Piau, N. El Kissi and B. Tremblay, J. Non-Newtonian Fluid Mech. 34, 145-180 (1990).

5. L. Robert, B. Vergnes and Y. Demay, J. Non-Newtonian Fluid Mech. 112, 27-42 (2003).

6. H. Kramer and J. Meissner, Proceedings of the 8th International Congress on Rheology 2, Naples, 463 (1980).

7. M. R. Mackley and I. P. T. Moore, J. Non-Newtonian Fluid Mech. 21, 337-358 (1986).

8. E. Wassner, M. Schmidt and H. Münstedt, J. Rheol. 43, 1339-1353 (1999).

9. M. Schmidt, E. Wassner and H. Münstedt, Mech. Time-Depend. Mater. 3, 371-393 (1999).

10. D. Hertel, R. Valette and H. Münstedt, J. Non-Newtonian Fluid Mech. 153, 82-94 (2008).

11. F. Durst and J. H. Whitelaw, Principles and Practice of Laser-Doppler-Anemometry, New York: Academic Press, 1981.

12. D. Hertel and H. Münstedt, J. Non-Newtonian Fluid Mech. 153, 73-81 (2008).

13. T. Gough et al., Plastics, Rubber and Composites 37, 158-165 (2008).

14. H. Janeschitz-Kriegel, Polymer Melt Rheology and Flow Birefringence, New York: SpringerVerlag, 1983.

15. G. G. Fuller, Optical Rheometry of Complex Fluids, New York: Oxford University Press, 1995.

16. M. T. Martyn, C. Nakason and P. D. Coates, J. Non-Newtonian Fluid Mech. 91, 109-122 (2000).

17. M. T. Martyn, C. Nakason and P. D. Coates, J. Non-Newtonian Fluid Mech. 91, 123-142 (2000). 
Copyright of AIP Conference Proceedings is the property of American Institute of Physics and its content may not be copied or emailed to multiple sites or posted to a listserv without the copyright holder's express written permission. However, users may print, download, or email articles for individual use. 